\title{
Doença de Creutzfeldt-Jakob, Variante de Heidenhain. Relato de Caso e Revisão do Tema.
}

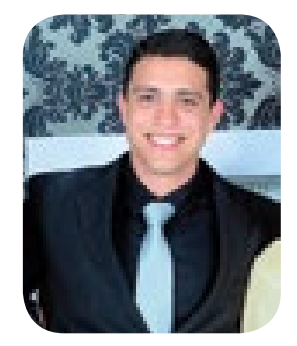

\author{
Matheus Mendes Pires ${ }^{1}$, Itana Naiara Costa Ribeiro', Tiago Santana \\ Santos Queiroz', Jamile Seixas Fukuda ${ }^{1}$, Thiago Fukuda', \\ Pedro Antonio Pereira de Jesus ${ }^{1}$
}

\section{RESUMO}

A doença de Creutzfeldt-Jakob (DCJ) é uma rara desordem neurodegenerativa, fazendo parte do espectro das doenças priônicas. Seu quadro típico é o de demência rapidamente progressiva, associada a sintomas cerebelares e mioclonias, com desfecho de morte ocorrendo em média de um ano. Em raros casos, sintomas visuais podem estar presentes, caracterizando a variante de Heidenhain. Descrevemos o caso de uma mulher de 62 anos, internada em nosso serviço com o quadro de demência avançada instalada no período de 45 dias, associada inicialmente à ataxia de marcha, alucinações visuais, mioclonias e posterior mutismo acinético. Realizada investigação etiológica do quadro, com evidência em ressonância magnética (RM) de crânio e eletroencefalograma (EEG) de alterações típicas da DCJ, com o diagnóstico provável da variante de Heidenhain da DCJ, realizado baseado nos critérios do Centers for Disease Controland Prevention - CDC.

PALAVRAS-CHAVE: doença de Creutzfeldt-Jakob; variante de Heidenhain; doença priônica; demência rapidamente progressiva.

KEY WORDS: Creutzfeldt-Jakob disease, Heidenhain variant, priondisease, rapidly progressive dementias.

\section{RELATO DE CASO}

V.S.J., 62 anos, negra, sem comorbidades prévias conhecidas, com história de em meados de dezembro/2015 iniciou quadro caracterizado como desequilíbrio de marcha, assemelhando-se a indivíduo alcoolizado (necessidade de apoiar-se nas paredes, episódios de queda), associado à cefaleia holocraniana mal caracterizada. Posteriormente apresentou queixa de alterações visuais, descritas como alucinações (via objetos e indivíduos inexistentes), com posterior diminuição de acuidade visual, associado a quadro demencial de rápida progressão.

A paciente evoluiu com declínio cognitivo importante, tornando-se acamada, sem se comunicar, com grande dificuldade de deglutição, apresentando também episódios de abalos musculares involuntários, especialmente no membro superior direito, desencadeados por sons altos no ambiente. A paciente foi vista em outro serviço e diagnosticada como portadora de transtorno depressivo grave, sendo então institucionalizada em casa de repouso.

Após 45 dias de início dos sintomas, a paciente foi encaminhada para nosso serviço, em função de febre e relato de piúria, sendo então contactada a equipe de Neurologia para melhor elucidação do caso. Os familiares negaram casos semelhantes na família ou contato com indivíduos com quadro semelhante.

Além da história já descrita, o exame físico da paciente evidenciava um estado de mutismo acinético, emagrecimento, força muscular grau 3 globalmente, sem assimetrias, hiperreflexia global, com sinal de Babinski bilateral. Era notável a presença de mioclonias espontâneas em região apendicular, mais intenso no membro superior direito, com startle sonoro e tátil presentes.

Em virtude da necessidade de investigação do quadro de demência rapidamente progressiva, a paciente foi internada em nosso serviço. $O$ fato da paciente apresentar quadro de demência rapidamente progressiva associada aos sinais e sintomas relatados, em especial as mioclonias e alterações visuais, foi aventada como principal suspeita diagnóstica Creutzfeldt Jakob variante de Heidenhain. $\mathrm{Na}$ investigação da paciente foi realizado o estudo do líquido cefalorraquidiano (LCR), ressonância magnética (RM) do crânio, eletroencefalograma (EEG), tomografia computadorizada (TC) de tórax e abdome. Em paralelo à investigação 
diagnóstica, foi iniciado suporte nutricional e familiar, além de tratamento de infecção urinária vigente.

Os resultados dos exames nos possibilitaram a exclusão de doenças paraneoplásicas, alem de realização do diagnóstico provável para a forma esporádica da doença de Creutzreldt Jakob, variante de Heidenhain, pois o EEG e RM de crânio apresentavam padrão típico da doença (Figura 1), alem da associação do quadro clínico. A pesquisa da proteína 14.3.3 no LCR aguardava realização.

Com a realização do diagnóstico provável, os familiares foram abordados do prognóstico sombrio da paciente, com a posterior aceitação de priorização de
medidas de conforto para a mesma.

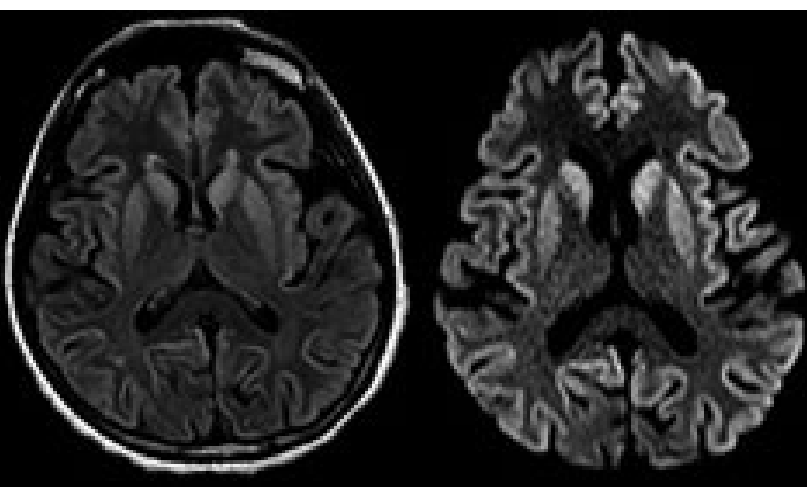

Figura 1 - Imagens do exame de ressonância magnética. A imagem mostra alteração de sinal nas sequências (ât dire evidenciando hipersinal em núcleos de base e córtex, com restrição à difusão no DWI.

\section{DISCUSSÃo}

As doenças priônicas são um grupo de doenças neurodegenerativas, causadas por uma proteina infectante, o prion, ocorrendo em sua grande maioria das vezes de forma esporádica $(85 \%)$, podendo também ser de cunho genético (10-15\%) ou adquirido $(1-5 \%)^{1,2,5}$

A doença de Creutzfeldt-Jakob esporádica (DCJe) possui a incidencia de 1 a 1.5 de casos/milhão por ano, com uma média de idade em torno dos 60 anos, existindo ampla variação na literatura (12 - 98 anos). É rara em indivíduos abaixo dos 30 anos, não possuindo predileção por gêneros ${ }^{1,2,5}$.

A sintomatologia tem grande variação, usualmente instalada de maneira subaguda, com raros casos relatados de início agudo. O transtorno cognitivo é sintoma mais prevalente na doença $(40 \%)$, usualmente ocorrendo de forma rapidamente progressiva, com mplo dano cognitivo (alteração de memória, disfunção executiva e distúrbio de linguagem $)^{1,2,10}$. Sintomas cerebelares também são encontrados, usualmente associados à queixa de desequilíbrio, caracterizados como ataxia da marcha. As mioclonias, espasmos musculares breves, involuntários, focais ou generalizados estão presentes em $90 \%$ dos pacientes, podendo estar associados a estímulos sonoros ou táteis (startle). Outros sintomas são a alteração do humor (depressão, irritabilidade) e alteraçoes visuais, estas mais raras, podendo ser caracterizadas como diplopia, turvação visual, ou ate mesmo como alucinações visuais complexas, que, quando presentes, caracterizam a variante de Heidenhain ${ }^{6,7}$.

O diagnóstico definitivo da doença é feito através da biópsia cerebral do paciente, entretanto, em função do risco de infectividade com o manuseio de material do sistema nervoso central dos pacientes (especialmente meninges, tecido cerebral, líquor e córnea), utilizam-se os critérios diagnósticos do Centers for Disease Controland Prevention - CDC (Quadro 1).

Os achados na RM de crânio podem ser vistos no período inicial da doença, porém, em alguns casos, só aparecerão com a repetição do exame em fase mais avançada. A alteração mais característica (sensibilidade de 91-96\%) consiste na restrição à difusão, caracterizada como hipersinal na sequência DWI, observada nos giros corticais e em núcleos da base, sendo as anormalidades na substância branca tipicamente ausentes em tais pacientes. $O$ estudo do líquor usualmente está inalterado, podendo apresentar discreta hiperproteinorraquia ${ }^{3,5,9,10}$. Um marcador de que deve ser solicitado em todo caso suspeito de DCJ e a proteina 14.3.3, com sensibilidade de cerca de $90 \%$. Outro exame complementar que auxilia no diagnostico e o EEG, com seu achado típico (complexo ponta-onda periódico) presente em $2 / 3$ dos pacientes ${ }^{4,5,8,11}$. Devem ser investigadas outras possibilidades diagnósticas juntamente com a da DCJ, sendo necessária a exclusão de doenças paraneoplásicas e encefalites autoimunes, os principais diagnosticos diferenciais da doença.

o paciente usualmente evolui com o desfecho de morte em cerca de 1 ano em $90 \%$ dos casos, usualmente de complicações infecciosas. Atualmente não existem tratamentos especificos para a DCJ. O suporte familiar e indispensavel, bem como o esclarecimento de informações pertinentes sobre a doença e explcação do prognóstico sombrio atrelado ao diagnóstico, sendo o trabalho da equipe multiprofissional e da equipe de Cuidados Paliativos imprescindivel.
Quadro 1. Doencas, sinais e sintomas facciosos descritos em casos de TFIO

\begin{tabular}{|c|c|}
\hline \multicolumn{2}{|c|}{$\begin{array}{l}\text { Critérios diagnósticos para Creutzfeldd-Jakob esporádica do Centers for } \\
\text { Disease Control and Prevention }\end{array}$} \\
\hline Definitivo & $\begin{array}{l}\text { Detecção de proteína priônica protease-resistente ou } \\
\text { fibrilas associadas a a scrapiena neuropatolologia, técnica } \\
\text { de imunoensaio e/ou Western Blot. }\end{array}$ \\
\hline Provável & $\begin{array}{l}\text { Sem achados de diagnósticos alternativos e demênncia } \\
\text { progressiva com pelo menos } 2 \text { (i) - (iv) e um de (a) - (c). }\end{array}$ \\
\hline Possivel & $\begin{array}{l}\text { Sem achados de diagnósticos alternativos e demência } \\
\text { progressiva menos de } 2 \text { anos e pelo menos } 2 \text { de (i) - (iv) } \\
\text { e um de (a) - (c). }\end{array}$ \\
\hline \multicolumn{2}{|c|}{ 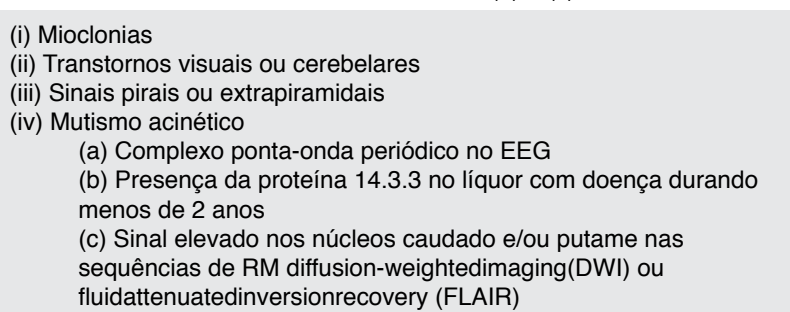 } \\
\hline
\end{tabular}

\section{REFERÊNCIAS}

Vitali P, Maccagnano E, Caverzasi E, et al Diffusion-weighted MRI hyperintensity patterns differentiate CJD from other rapid dementias. Neurology 2011;76:1711-1719.

2. Takada LT1, Geschwind MD. Prion Diseases. Semin Neurol. 2013 Sep;33(4):348-56. doi: 10.1055 s-0033-1359314. Epub 2013 Nov 14

3. Kojima, Gotaro et al. "Creutzfeldt-Jakob Disease: A Case Report and Differential Diagnoses. Hawai'i Journal of Medicine \& Public Health 72.4 (2013): 136-139. Print.

4. Geschwind MD, Haman A, Miller BL. Rapidly progressive dementia. NeurolClin 2007;25:783-807. 5. Geschwind MD. Clinical trials for prion disease difficult challenges, but hope for the future. Lancet Neurol 2009;8:304-306.

6. Geschwind MD. Dementia: rapidly progressive dementia: prion diseases and other rapid dementias. Continuum 2010;16:153-175.

7. Green A, Sanchez-Juan P, Ladogana A, et al. CSF analysis in patients with sporadic CJD and other transmissible spongiform encephalopathies. Eur J Neurol 2007;14:121-124.

8. Patrícia, Coral, Germiniani, Francisco M B., \& Silvado, Carlos E.. (2005). Creutzfeldt-Jakob'sdisease: case reportwithemphasisontheelectroencephalographicfeatures. JournalofEpilepsyandClinicalNeurophysiology, 11(4), 189-192.

9. Arruda, Walter Oleschko, Bordignon, Kelly C.
Milano Jerônimo B \&Ramina Ricardo (2004) Doença de Creutzfeldt-Jakob forma Heidenhain: relato de caso com achados de ressonância magnética 10. Arquivos de Neuropsiquiatria, 62(2a), 347-352. 10. Meissner B, Kallenberg K, Sanchez-Juan $P$, et al. MRI lesion profiles in sporadic Creutzfeld-Jakob disease. Neurology 2009;72:1994-2001. 11. Steinhof BJ, Zerr I, Glatting M, Schulz-Schaeffer W, Poser S, Kretzschmar HA. Diagnostic value of periodic complexes in Creutzfeldt-Jakob disease.
Ann Neurol 2004:56:702-708.

1- Serviço de Neurologia Clínica do Hospital Santa Izabel

Endereço para correspondência:

matheusmpires@yahoo.com.br 